\title{
MINERALIZAÇÃO DE NITROGÊNIO EM LATOSSOLOS ADUBADOS COM RESÍDUOS ORGÂNICOS ${ }^{(1)}$
}

\author{
Waldete Japiassu de Oliveira Carneiro ${ }^{(2)}$, Carlos Alberto Silva ${ }^{(3)}$, Joel Augusto Muniz ${ }^{(4)}$ \& \\ Taciana Villela Savian ${ }^{(5)}$
}

\begin{abstract}
RESUMO
A taxa de mineralização do nitrogênio (N) varia de um resíduo orgânico para outro, evidenciando-se dependente da sua composição química e interação do material orgânico com o solo. Este estudo foi realizado com o objetivo de avaliar a dinâmica de mineralização de $\mathrm{N}$ de resíduos orgânicos incubados em Latossolos. $\mathrm{O}$ experimento foi conduzido no período de julho de 2007 a abril de 2008, sendo incubadas amostras de estercos, lodos, compostos, substrato e turfa em areia lavada, Latossolo Vermelho-Amarelo (LVA) e Latossolo Vermelho distroférrico (LVdf), com 240 e $670 \mathrm{~g} \mathrm{~kg}^{-1}$ de argila, respectivamente. $\mathrm{O} \mathrm{N}$ mineralizado foi avaliado medindo-se os teores de $\mathrm{N}-\mathrm{NH}_{4}{ }^{+}$e $\mathrm{N}-\mathrm{NO}_{3}{ }^{-}$em lixiviados coletados aos $15,30,45,60$, $75,90,120,150,180,210,240$ e 270 dias de incubação. A mineralização de $N$ ocorreu a maiores taxas nos períodos iniciais de incubação. $\mathrm{O} \mathrm{N}$ mineralizado (33 a 199,2 $\mathrm{mg} \mathrm{kg}^{-1}$ ) após 270 dias de incubação é regulado pelos teores de $\mathbf{N}$ total e de carbono solúvel em água dos resíduos orgânicos. Independentemente do meio utilizado para incubação, os estercos de galinha e de codorna propiciaram os maiores teores de $\mathbf{N}$ mineralizado nos Latossolos. À exceção dos estercos de galinha e codorna, há imobilização líquida de N no LVA; mineralização líquida de N é verificada no LVdf, para todos os resíduos orgânicos. Em relação às formas de $\mathrm{N}$ disponibilizadas pelos resíduos, predomina o $\mathrm{N}$-nitrato, sendo a proporção $\mathrm{N}$-nítrico/ $\mathrm{N}$-amoniacal dependente da mistura solo-resíduo estudada.
\end{abstract}

Termos de indexação: $N$ mineral, matéria orgânica, imobilização, nitrificação, grau de humificação.

(1) Recebido para publicação em 21 de dezembro de 2011 e aprovado em 11 de março de 2013.

(2) Professora Adjunta, Faculdade de Ciência e Tecnologia - Área 1. CEP 40279-150 Salvador (BA), Brasil. E-mail: wcarneiro@area1.edu.br

(3) Professor Associado, Departamento de Ciência do Solo, Universidade Federal de Lavras - UFLA. Caixa Postal 3037. CEP 37200-000 Lavras (MG), Brasil. E-mail: csilva@dcs.ufla.br

(4) Professor Titular, Departamento de Ciências Exatas, UFLA. E-mail: joamuniz@dex.ufla.br

(5) Professora, Departamento de Ciências Exatas. Universidade de São Paulo/ESALQ. Av. Pádua Dias, 11. CEP 13418-900 Piracicaba (SP), Brasil. E-mail: tvsavian@usp.br 


\title{
SUMMARY:NITROGEN MINERALIZATION IN LATOSOLS FERTILIZED WITH ORGANIC RESIDUES
}

\begin{abstract}
The $N$ mineralization rate varies from one organic residue to another, and depends on the chemical composition of a residue and its interaction with the soil. This study assessed the dynamics of $N$ mineralization of organic residues incubated in Oxisols. The experiment was carried out from July 2007 to April 2008. Samples of manures, sludges, composts, peat, and substrate were incubated in washed sand, in a Red Yellow Latosol (RYL), and in a Red Latosol (RL), with 240 and $670 \mathrm{~g} \mathrm{~kg}^{-1}$ of clay, respectively. Mineralized $\mathrm{N}$ was assessed by measuring the contents of $\mathrm{N}_{-} \mathrm{NH}_{4}{ }^{+}$and $\mathrm{N}_{-} \mathrm{NO}_{3}-$ in leachates (after 15, 30, 45, 60, 75, 90, 120, 150, 180, 210,240 , and 270 days of incubation). $N$ mineralization occurs at higher rates in the initial periods of incubation and the mineralized $N\left(33-199.2 \mathrm{mg} \mathrm{kg}^{-1}\right)$ after 270 days is regulated by the total $N$ and water-soluble carbon contents in organic residue. Regardless of the medium used for incubation, chicken and quail manures resulted in the highest levels of mineralized $N$ in both Oxisols. Aside from quail and chicken manures, the other fertilizers resulted in net immobilization of $N$ in the $R Y L$; all organic residues studied resulted in net $N$ mineralization in the RL. Nitrate is the main $N$ form released by the organic residues; the proportion $N$ nitrate / $N$-ammonium depends on the soil-residue mixture.
\end{abstract}

Index terms: Mineral $N$, organic matter, immobilization, nitrification, humification degree.

\section{INTRODUÇÃO}

No Brasil, há grande oferta de resíduos orgânicos, que são fontes de $\mathrm{C}, \mathrm{N}$ e de outros nutrientes para as plantas. O uso racional dos resíduos orgânicos implica, entre outros aspectos, na definição correta da dose a ser aplicada nas lavouras. No caso do N, o cálculo da dose mostra-se dependente da dinâmica de mineralização do nutriente, que é influenciada pela composição química dos resíduos e por fatores bióticos e abióticos associados aos solos, onde esses materiais são aplicados (Bernal et al., 1998; Trinsoutrot et al., 2000; Khalil et al., 2005).

Os adubos orgânicos utilizados como fontes de $\mathrm{N}$ nas lavouras englobam os estercos de bovinos, suínos e galinha, tortas vegetais, lodo de esgoto, torta de filtro, camas aviárias e compostos orgânicos, produzidos a partir de restos vegetais ou do lixo urbano. Em geral, os teores de $\mathrm{N}$ nesses fertilizantes são variáveis, na faixa de 0,6 a 4,6\% (Melo et al., 2008a) e de 0,05 a $4,9 \%$ (Higashikawa et al., 2010); teores mais elevados de $\mathrm{N}$ são verificados em tortas de semente de amendoim, mamona e algodão, que possuem de 4 a $7 \%$ de N (Kiehl, 1985; Costa et al., 2004). Além desses materiais, o lodo pode constituir-se em outra fonte de $\mathrm{N}$ para as plantas, por apresentar teores de $\mathrm{N}$ de até $8 \%$, muito embora, para diferentes lodos, a fração de mineralização do N orgânico, em 105 dias de incubação, possa variar de 20 a $38 \%$ (Boeira et al., 2002). Segundo Amlinger et al. (2003), a mineralização de N orgânico de compostos, entre outros fatores, é regulada pela relação $\mathrm{C} / \mathrm{N}$ das matérias-primas utilizadas na compostagem e pelas frações de $\mathrm{C}$ e $\mathrm{N}$ presentes nos compostos e sujeitas à decomposição.

Em razão da grande variedade de resíduos disponíveis para uso como fontes de $\mathrm{N}$, aliada às diferenças na composição química e no grau de humificação desses materiais, é razoável supor que as frações de $\mathrm{N}$ nos resíduos e as suas taxas de mineralização variem de um material para outro, conforme afirmaram Trinsoutrot et al. (2000). Segundo esses autores, o processo de mineralização, entre outros fatores, é regulado pela composição química dos resíduos; no caso dos lodos de esgoto, a quantidade de $\mathrm{N}$ mineralizada é regulada pelo material de origem e pelo processo de tratamento a que o lodo é submetido (Boeira et al., 2002). Segundo Janzen \& Kucey (1988), a composição bioquímica dos resíduos vegetais explica menos a mineralização de $\mathrm{N}$ do que a concentração de $\mathrm{N}$ no tecido vegetal. Para adubos verdes do semiárido, Alves et al. (2011) verificaram que houve ampla variação no $\mathrm{N}$ mineralizado e que a magnitude da relação polifenol/ $\mathrm{N}$ foi determinante para a prevalência da mineralização ou da imobilização de N. Para lodos de esgoto, a presença de metais pesados tanto pode aumentar quanto limitar a mineralização de $\mathrm{N}$, de modo que mais pesquisa é necessária para elucidar esses comportamentos conflitantes (Dar \& Mishra, 1994; Khan \& Scullion, 2002; Cabrera et al., 2005).

As características físico-químicas do solo são de grande importância na mineralização do $\mathrm{N}$, em que o resíduo é aplicado, uma vez que ao ser adicionado ao solo os resíduos orgânicos interagem com essa matriz (Aita \& Giacomini, 2007). As taxas e quantidades de $\mathrm{N}$ mineralizadas em solos são dependentes, entre outros fatores, do grau de fertilidade, da textura do solo, do grau de acidez, dos teores de $\mathrm{Ce} \mathrm{Ne}$ do balanço dos processos de perda e de ganhos de $\mathrm{N}$ mineral do sistema solo-planta (Cabrera et al., 2005; Khalil et al., 2005; Schomberg et al., 2009).

Muitos trabalhos têm sido realizados visando elucidar questões relacionadas à dinâmica de mineralização do $\mathrm{N}$ por resíduos orgânicos (Monteiro et al., 2002; Aita \& Giacomini, 2003; Mantovani et 
al., 2006.; Espindola et al., 2006; Alcântara et al., 2007); contudo, os trabalhos publicados no Brasil, até o momento, concentraram-se em aspectos relacionados à mineralização de $\mathrm{N}$ de restos culturais. Pouco se sabe sobre a dinâmica de mineralização de $\mathrm{N}$ para os resíduos de origem animal e agroindustrial. Os teores de $\mathrm{N}$ total, as formas de $\mathrm{N}$ e o grau de humificação dos resíduos orgânicos podem condicionar diferentes velocidades de mineralização e quantidades acumuladas de $\mathrm{N}$ mineralizado. É bastante provável que as maiores taxas de mineralização ocorram nos períodos iniciais de incubação e que os resíduos mais ricos em $\mathrm{N}$ e menos humificados liberem maiores quantidades de $\mathrm{N}$ do que os mais pobres e menos lábeis.

Objetivou-se avaliar a dinâmica de mineralização do nitrogênio por resíduos orgânicos incubados em amostras de Latossolos com características químicas e textura contrastantes.

\section{MATERIAL E MÉTODOS}

O experimento foi conduzido de julho de 2007 a abril de 2008. Foram utilizadas amostras da camada superficial $(0-20 \mathrm{~cm})$ de um Latossolo Vermelho distroférrico e de um Latossolo Vermelho-Amarelo com as seguintes características: $240 \mathrm{~g} \mathrm{~kg}^{-1}$ de argila (textura média); $\mathrm{pH}$ em água $=5,4 ; \mathrm{Al}^{3+}=0,2 \mathrm{cmol}_{\mathrm{c}}$ $\mathrm{dm}^{-3} ; \mathrm{Ca}^{2+}=0,4 \mathrm{cmol}_{\mathrm{c}} \mathrm{dm}^{-3} ; \mathrm{Mg}^{2+}=0,2 \mathrm{cmol}_{\mathrm{c}} \mathrm{dm}^{-3} ;$ $\mathrm{P}=0,4 \mathrm{mg} \mathrm{dm}^{-3} ; \mathrm{K}^{+}=20 \mathrm{mg} \mathrm{dm}{ }^{-3} ; \mathrm{MO}=15,5 \mathrm{~g} \mathrm{~kg}^{-1}$ (Latossolo Vermelho-Amarelo) e $670 \mathrm{~g} \mathrm{~kg}^{-1} \mathrm{de}$ argila (textura muito argilosa); $\mathrm{pH}$ em água $=4,3$; $\mathrm{Al}^{3+}=1,4 \mathrm{cmol}_{\mathrm{c}} \mathrm{dm}^{-3} ; \mathrm{Ca}^{2+}=1,0 \mathrm{cmol}_{\mathrm{c}} \mathrm{dm}^{-3} ; \mathrm{Mg}^{2+}$ $=0,4 \mathrm{cmol}_{\mathrm{c}} \mathrm{dm}^{-3} ; \mathrm{P}=2,3 \mathrm{mg} \mathrm{dm}{ }^{-3} ; \mathrm{K}^{+}=53 \mathrm{mg} \mathrm{dm}$; e $\mathrm{MO}=33 \mathrm{~g} \mathrm{~kg}^{-1}$ (Latossolo Vermelho distroférrico), conforme métodos de análise descritos por Silva (1999). A areia lavada apresentou as seguintes características: $\mathrm{pH}$ em água $=6,2 ; \mathrm{MO}=1,3 \mathrm{~g} \mathrm{~kg}^{-1} ; \mathrm{K}^{+}=3 \mathrm{mg} \mathrm{kg}^{-1} ; \mathrm{e}$ $\mathrm{P}=1 \mathrm{mg} \mathrm{kg}^{-1}$, conforme protocolos analíticos descritos em Silva (1999). Na areia, os teores de $\mathrm{N}$ total, $\mathrm{Ca}^{2+}$, $\mathrm{Al}^{3+} \mathrm{e} \mathrm{Mg}^{2+}$ ficaram abaixo do limite de detecção das técnicas analíticas utilizadas para caracterização da fertilidade do solo. A areia foi coletada no município de Itutinga, MG, e submetida a várias lavagens com solução ácida de $\mathrm{HCl}$ diluído em água, na proporção de 1:1; em seguida, foi lavada, repetidas vezes, com água desmineralizada, a fim de retirar nutrientes a ela associados.

Os materiais orgânicos foram coletados em municípios do sul de Minas Gerais, sendo analisados os seguintes resíduos: amostras de lodo de esgoto (lodos 1 e 2), oriundas das estações de tratamento de esgoto de duas cidades de médio porte, localizadas em municípios de Minas Gerais; esterco de galinha; composto orgânico; esterco de suíno; esterco de codorna; esterco de bovino; substrato orgânico; esterco de equino; e composto de lixo e turfa. Após a etapa de coleta, as amostras foram secas $\left(70^{\circ} \mathrm{C}\right)$, peneiradas $(>0,25 \mathrm{~mm})$ e armazenadas em dessecador, para posterior análise.
A caracterização físico-química de parte desses resíduos consta em Melo et al. (2008b) e é apresentada no quadro 1.

A dose de resíduo orgânico aplicada ao solo visou suprir $100 \mathrm{mg} \mathrm{kg}^{-1}$ de $\mathrm{N}$, quantidade suficiente para o crescimento pleno da maioria das culturas. $\mathrm{O}$ cálculo foi feito com base nos teores total e disponível de $\mathrm{N}$ nos materiais orgânicos (Quadro 2) e no teor de água de cada resíduo orgânico. As quantidades dos resíduos adicionadas em cada parcela experimental foram: lodo $1,1,72 \mathrm{~g} \mathrm{~kg}^{-1}$; lodo $2,5,08 \mathrm{~g} \mathrm{~kg}^{-1}$; esterco de galinha, $0,90 \mathrm{~g} \mathrm{~kg}^{-1}$; composto de lixo, $1,86 \mathrm{~g} \mathrm{~kg}^{-1}$; composto orgânico, 3,54 $\mathrm{g} \mathrm{kg}^{-1}$; esterco de suíno, 2,16 g kg-1; esterco de codorna, $1,28 \mathrm{~g} \mathrm{~kg}^{-1}$; esterco de equino, $2,68 \mathrm{~g} \mathrm{~kg}^{-1}$; esterco de bovino, $3,74 \mathrm{~g} \mathrm{~kg}^{-1}$; substrato orgânico, 8,12 $\mathrm{g} \mathrm{kg}^{-1}$; e turfa, $7,18 \mathrm{~g} \mathrm{~kg}^{-1}$.

O delineamento utilizado foi o de blocos ao acaso, em esquema fatorial [(11 x 3) + 2], ou seja, foram estudados 11 resíduos orgânicos incubados em dois solos (LVdf e LVA) e em areia lavada, mais um tratamento adicional, constituído por parcelas do LVdf e LVA incubadas sem a adição de resíduo orgânico, em três repetições, totalizando 105 parcelas experimentais.

Foram incubadas $200 \mathrm{~g}$ de solo e areia lavada por 270 dias, em colunas de PVC com $20 \mathrm{~cm}$ de altura e diâmetro de $75 \mathrm{~mm}$, que foram fixadas em bancadas de madeira. Antes do início da incubação, a acidez das amostras dos solos foi corrigida visando atingir saturação por bases igual a $60 \%$, utilizando-se sais p.a. de $\mathrm{CaCO}_{3}$ e $\mathrm{MgCO}_{3}$, na proporção de 4:1, respectivamente. Aumidade das amostras foi mantida próxima a $70 \%$ da capacidade de campo dos solos, por meio de pesagens diárias das amostras incubadas. A temperatura média ao longo do período de incubação foi de $24{ }^{\circ} \mathrm{C}$, com variação média de $\pm 4^{\circ} \mathrm{C}$.

A avaliação da mineralização do $\mathrm{N}$ orgânico dos resíduos foi realizada por meio da medição do teor de $\mathrm{N}$ mineral acumulado $\left(\mathrm{N}-\mathrm{NH}_{4}{ }^{+}\right.$e N-NO ${ }_{3}^{-}$), sendo as coletas de lixiviados efetuadas aos $15,30,45,60,75$, $90,120,150,180,210,240$ e 270 dias, após o início da incubação. Nessa etapa de lavagem das amostras de solo/resíduos incubadas, foi utilizada solução de $\mathrm{CaCl}_{2}$ $0,01 \mathrm{~mol} \mathrm{~L}^{-1}$. O lixiviado foi coletado em recipientes posicionados abaixo de cada coluna de PVC e teve seu volume medido, sendo, em seguida, filtrado (filtro com poro de diâmetro de $0,45 \mu \mathrm{m}$ ) e armazenado em refrigerador $\left(4^{\circ} \mathrm{C}\right)$, para posterior análise do teor de $\mathrm{N}$ mineralizado nas formas de $\mathrm{NH}_{4}{ }^{+} \mathrm{e} \mathrm{NO}_{3}$; utilizouse, para isso, destilador de arraste de vapores (método semimicro Kjeldahl) (Tedesco et al., 1995).

A taxa de mineralização diária do $\mathrm{N}$ foi obtida pela divisão do $\mathrm{N}$ mineralizado em cada período de incubação pelos respectivos números de dias de incubação.

Às quantidades acumuladas de $\mathrm{N}$ mineralizado foi ajustado modelo de cinética de primeira ordem, proposto por Stanford \& Smith (1972), conforme equação mostrada a seguir: 
Quadro 1. Teores totais de nitrogênio ( $\mathrm{N}$ total), carbono solúvel em água (CSA), carbono lábil (C-lábil), carbono total ( $\mathrm{C}$ total) e relação $\mathrm{C} / \mathrm{N}$ dos resíduos orgânicos estudados

\begin{tabular}{|c|c|c|c|c|c|}
\hline Resíduo & $\mathbf{N}$ total & CSA & C-lábil & C total & Relação C/N \\
\hline & dag $\mathrm{kg}^{-1}$ & $\mathrm{mg} \mathrm{kg}^{-1}$ & $\mathrm{~g} \mathrm{~kg}^{-1}$ & dag $\mathrm{kg}^{-1}$ & \\
\hline Lodo de esgoto 1 & 2,5 & 246 & 8,9 & 21,7 & 8,7 \\
\hline Lodo de esgoto 2 & 0,8 & 26 & 2,3 & 5,6 & 7,0 \\
\hline Esterco de galinha & 4,6 & 1181 & 4,1 & 19,2 & 4,2 \\
\hline Composto orgânico & 1,2 & 316 & 4,5 & 13,6 & 11,3 \\
\hline Esterco de suíno & 1,9 & 131 & 6,1 & 22,8 & 12,0 \\
\hline Esterco de codorna & 3,3 & 585 & 5,5 & 22,7 & 6,9 \\
\hline Esterco de bovino & 1,1 & 453 & 4,9 & 12,2 & 11,1 \\
\hline Substrato orgânico & 0,6 & 42 & 9,5 & 31,3 & 52,2 \\
\hline Composto de lixo & 2,7 & 450 & 2,7 & 18,8 & 7,0 \\
\hline Turfa & 0,8 & 20 & 0,8 & 9,4 & 11,7 \\
\hline Esterco de equino & 1,8 & 400 & 2,9 & 39,4 & 21,9 \\
\hline
\end{tabular}

Fonte: Parte dos dados foi compilada de Melo et al. (2008b).

$$
N m=N o[1-\exp (-c t)]+\varepsilon
$$

em que $\mathrm{Nm}$ é $\mathrm{N}$ mineralizado, em $\mathrm{mg} \mathrm{kg}^{-1} ;$ No, o potencial de mineralização de $\mathrm{N}$, em $\mathrm{mg} \mathrm{kg}^{-1} ; c$, taxa ou constante de mineralização do nutriente, $\mathrm{em} \mathrm{mg} \mathrm{kg}^{-1} \mathrm{dia}^{-1}$; t, tempo de incubação, em dias; e $\varepsilon$, erro considerado aleatório, independente e identicamente distribuído de uma normal com média zero e variância constante $\sigma^{2}$. A escolha desse tipo de modelo foi feita em razão de ter sido essa a equação matemática que, em geral, melhor se ajustou ao conjunto de dados conseguidos, por causa dos coeficientes de determinação $\left(R^{2}\right)$ obtidos, que foram superiores a 0,9. Adicionalmente, Pereira et al. (2005) fizeram um estudo a respeito do ajuste de modelos não lineares para predizer a mineralização de $\mathrm{N}$ em Latossolo e concluíram que o modelo de Stanford \& Smith (1972), para situação de erros independentes, foi o que melhor se ajustou aos dados e que, além de apresentar elevados valores para o $R^{2}$, apresentou o menor valor para o erro de predição médio, entre os modelos estudados. Ainda, esse modelo apresentou apenas dois parâmetros que são biologicamente interpretáveis. Isso é uma vantagem em relação aos demais modelos não lineares, pois o aumento do número de parâmetros dificulta a convergência dos métodos iterativos, necessários ao processo de estimação dos parâmetros.

A partir dos valores da constante de mineralização de $\mathrm{N}$, calculou-se o tempo de meia-vida $\left(\mathrm{t}_{1 / 2}=\ln (2) / \mathrm{c}\right)$, cuja método de cálculo e dedução matemática utilizada para esse fim é apresentado em Paul \& Clark (1996). $\mathrm{O} \mathrm{t}_{1 / 2}$ corresponde ao tempo necessário para que metade do $\mathrm{N}$ mineralizado seja disponibilizada no solo. Os resultados foram submetidos à análise de variância e, ao verificar-se o efeito significativo (teste F), utilizouse o teste de Scott-Knott, a 1\%, para se compararem as médias de teores de $\mathrm{N}$ mineralizado líquido e de $\mathrm{N}$ mineral (nitrato e amônio) em solo; para isso, usou-se o programa SISVAR (Ferreira, 2003). Os teores de N mineralizado acumulado após 270 dias de incubação foram correlacionados com o $\mathrm{N}$ total, CSA, C-lábil e relação $\mathrm{C} / \mathrm{N}$ dos resíduos orgânicos.

\section{RESULTADOS E DISCUSSÃO}

Em relação à modelagem da dinâmica de mineralização de $\mathrm{N}$, o potencial de mineralização de $\mathrm{N}\left(39,8\right.$ a $\left.201,7 \mathrm{mg} \mathrm{kg}^{-1}\right)\left(N_{o}\right)$ e as taxas de mineralização do $\mathrm{N}(c)$ variaram entre os tratamentos, evidenciando-se dependentes do tipo de solo e do resíduo orgânico incubado (Quadro 2). Os maiores valores de $N_{o}$ foram verificados para os estercos de galinha e codorna e lodo de esgoto 1 , independentemente de os materiais terem sido incubados na areia ou em amostras de Latossolos. Foram verificados menores valores de No para o substrato e a turfa, no LVdf; substrato orgânico e esterco de equino, no LVA; e esterco de bovino, na areia. $\mathrm{O} N_{o}$ constitui-se em atributo importante, uma vez que pode se utilizado para predizer a disponibilidade de $\mathrm{N}$ em dado tempo e para estimar, junto com outros fatores, a absorção de $\mathrm{N}$ por plantas (Rhoden et al., 2006). As taxas de mineralização de $\mathrm{N}(c)$ variaram de 0,001 a 0,012 $\mathrm{dia}^{-1}$, com comportamento similar ao já destacado para o $N_{o}$.

As maiores quantidades de $\mathrm{N}$ mineralizado foram observadas para o LVdf, independentemente do resíduo analisado (Figura 1). Esse é o solo com maiores teores de matéria orgânica e de argila, de modo que, nele, o tratamento-controle (sem adição de resíduo) propiciou a obtenção de elevadas quantidades de $\mathrm{N}$, quase duas vezes a determinada para o tratamento-controle do LVA. Os trabalhos de Abreu Junior et al. (2002), Khalil et al. (2005) e Schomberg et al. (2009) ilustraram o papel exercido pelas características dos solos sobre a mineralização e disponibilidade de N. No LVdf, as maiores quantidades de $\mathrm{N}$ mineralizado acumulado 
foram notadas para os estercos de galinha e codorna. No LVdf, o $\mathrm{N}$ mineralizado até 270 dias variou de 124 (controle) a 199,2 (esterco de galinha) $\mathrm{mg} \mathrm{kg}^{-1}$. Em relação aos resíduos incubados nesse solo, a turfa e o substrato orgânico liberaram as menores quantidades de $\mathrm{N}$ mineral. Coincidentemente, a turfa e o substrato orgânico são os resíduos que apresentaram os menores teores de $\mathrm{N}$ total (Quadro 1). $\mathrm{Na}$ areia, não foi verificada diferença estatística entre o $\mathrm{N}$ mineralizado pelos resíduos orgânicos, mas foi notada tendência de maior liberação do nutriente, quando se incubaram os estercos de galinha e codorna. No LVA, a quantidade

Quadro 2. Estimativa dos parâmetros do modelo de Stanford \& Smith (1972) para os resíduos orgânicos incubados

\begin{tabular}{|c|c|c|c|}
\hline Resíduo orgânico & $\mathbf{N}_{\mathbf{0}}$ & c & $\mathbf{R}^{2}$ \\
\hline & $\mathrm{mg} \mathrm{kg}$ & $\operatorname{dia}^{-1}$ & \\
\hline \multicolumn{4}{|c|}{ Latossolo Vermelho distroférrico } \\
\hline Lodo de esgoto 1 & 190,3 & 0,0105 & 0,99 \\
\hline Lodo de esgoto 2 & 139,5 & 0,0120 & 0,95 \\
\hline Esterco de galinha & 201,7 & 0,0107 & 0,99 \\
\hline Composto de lixo & 169,2 & 0,0114 & 0,99 \\
\hline Composto orgânico & 167,9 & 0,0087 & 0,99 \\
\hline Esterco de suíno & 160,0 & 0,0075 & 0,99 \\
\hline Esterco de codorna & 191,7 & 0,0104 & 0,99 \\
\hline Esterco de bovino & 157,0 & 0,0104 & 0,96 \\
\hline Substrato orgânico & 146,2 & 0,0085 & 0,98 \\
\hline Turfa & 136,6 & 0,0116 & 0,98 \\
\hline Esterco de equino & 155,4 & 0,0143 & 0,98 \\
\hline \multicolumn{4}{|c|}{ Latossolo Vermelho-Amarelo } \\
\hline Lodo de esgoto 1 & 88,7 & 0,004 & 0,99 \\
\hline Lodo de esgoto 2 & 73,4 & 0,004 & 0,99 \\
\hline Esterco de galinha & 85,1 & 0,010 & 0,95 \\
\hline Composto de lixo & 50,2 & 0,001 & 0,99 \\
\hline Composto orgânico & 75,5 & 0,004 & 0,99 \\
\hline Esterco de suíno & 64,8 & 0,001 & 0,95 \\
\hline Esterco de codorna & 89,5 & 0,002 & 0,98 \\
\hline Esterco de bovino & 54,9 & 0,002 & 0,98 \\
\hline Substrato orgânico & 39,8 & 0,009 & 0,97 \\
\hline Turfa & 57,7 & 0,005 & 0,98 \\
\hline Esterco de equino & 44,8 & 0,002 & 0,90 \\
\hline \multicolumn{4}{|c|}{ Areia lavada } \\
\hline Lodo de esgoto 1 & 68,9 & 0,021 & 0,93 \\
\hline Lodo de esgoto 2 & 53,2 & 0,005 & 0,99 \\
\hline Esterco de galinha & 70,5 & 0,011 & 0,96 \\
\hline Composto de lixo & 59,7 & 0,006 & 0,99 \\
\hline Composto orgânico & 67,4 & 0,007 & 0,99 \\
\hline Esterco de suíno & 54,3 & 0,011 & 0,98 \\
\hline Esterco de codorna & 75,8 & 0,006 & 0,98 \\
\hline Esterco de bovino & 42,9 & 0,006 & 0,95 \\
\hline Substrato orgânico & 57,1 & 0,008 & 0,95 \\
\hline Turfa & 53,4 & 0,010 & 0,97 \\
\hline Esterco de equino & 56,4 & 0,016 & 0,91 \\
\hline
\end{tabular}

de $\mathrm{N}$ mineralizado pela incubação do esterco de galinha supera as demais produzidas pelos outros resíduos. Nesse solo, os estercos de suíno e o substrato orgânico disponibilizam as menores quantidades de $\mathrm{N}$ mineral, sendo notadas, também, para a maioria dos resíduos, quantidades de $\mathrm{N}$ mineralizado inferiores à do controle (LVA incubado sem resíduo). Evidenciaram-se, para as amostras de Latossolos, variações nas quantidades de $\mathrm{N}$ mineralizadas para os estercos. Segundo Eghball et al. (2002), essas diferenças em mineralização podem ser em razão do grau de estabilização química dos estercos, uma vez que os materiais compostados perdem, ao longo da compostagem, compostos mais lábeis, remanescendo nos estercos humificados compostos nitrogenados menos suscetíveis à decomposição.

As taxas de mineralização diária variaram de um resíduo para outro, apresentando-se dependentes da mistura solo-resíduo (Figura 2). Nos períodos iniciais de incubação, as taxas de mineralização de $\mathrm{N}$ verificadas para o LVdf superaram, para a maioria dos resíduos estudados, as determinadas para as amostras de LVA e areia. Foi elevada a velocidade de mineralização do $\mathrm{N}$ por alguns resíduos orgânicos estudados, nos períodos iniciais da incubação, sendo exemplos os estercos de galinha e codorna incubados no LVdf. À medida que o tempo de incubação aumentou, foi verificada estabilização e diminuição da taxa de mineralização. $\mathrm{O}$ tempo em que ocorreu a estabilização da taxa de mineralização variou de acordo com o solo e com o resíduo orgânico incubado. No LVdf, valores constantes de taxa de mineralização só foram verificados para tempos de incubação, na maioria dos casos, superiores a 90-210 dias. No LVA, a taxa de mineralização se estabilizou em menores patamares, em tempos de incubação inferiores a 90 dias, sendo exceção as taxas observadas para os estercos de galinha e codorna. A rápida mineralização do $\mathrm{N}$ no início da incubação e o decréscimo das taxas com o tempo foram relatados em diversos estudos (Boeira et al., 2002; Pansu \& Thuriés, 2003; Mantovani et al., 2006; Carneiro et al., 2007), atribuindo-se à qualidade e composição química dos resíduos orgânicos estudados; ou seja, no início da incubação são consumidas formas mais lábeis de $\mathrm{C}$, remanescendo no solo, à medida que avança o tempo de incubação, frações orgânicas menos suscetíveis à decomposição (Trinsoutrot et al., 2000; Pansu \& Thuriés, 2003). Segundo Giacomini \& Aita (2003), a maior parte do N mineralizado de resíduos culturais ocorreu nos primeiros 15 dias de incubação, sendo isso atribuído à decomposição de formas mais lábeis de $\mathrm{N}$, predominando, a posteriori, formas de $\mathrm{N}$ mais recalcitrantes. Para plantas de cobertura do cerrado, a maioria do $\mathrm{N}$ mineralizado foi disponibilizada no solo nos primeiros 42 dias de incubação (Torres et al., 2005).

A mineralização líquida (N-mineralização bruta $\mathrm{N}$ mineralizado no solo incubado sem adição de resíduo orgânico) variou muito de uma mistura solo-resíduo 


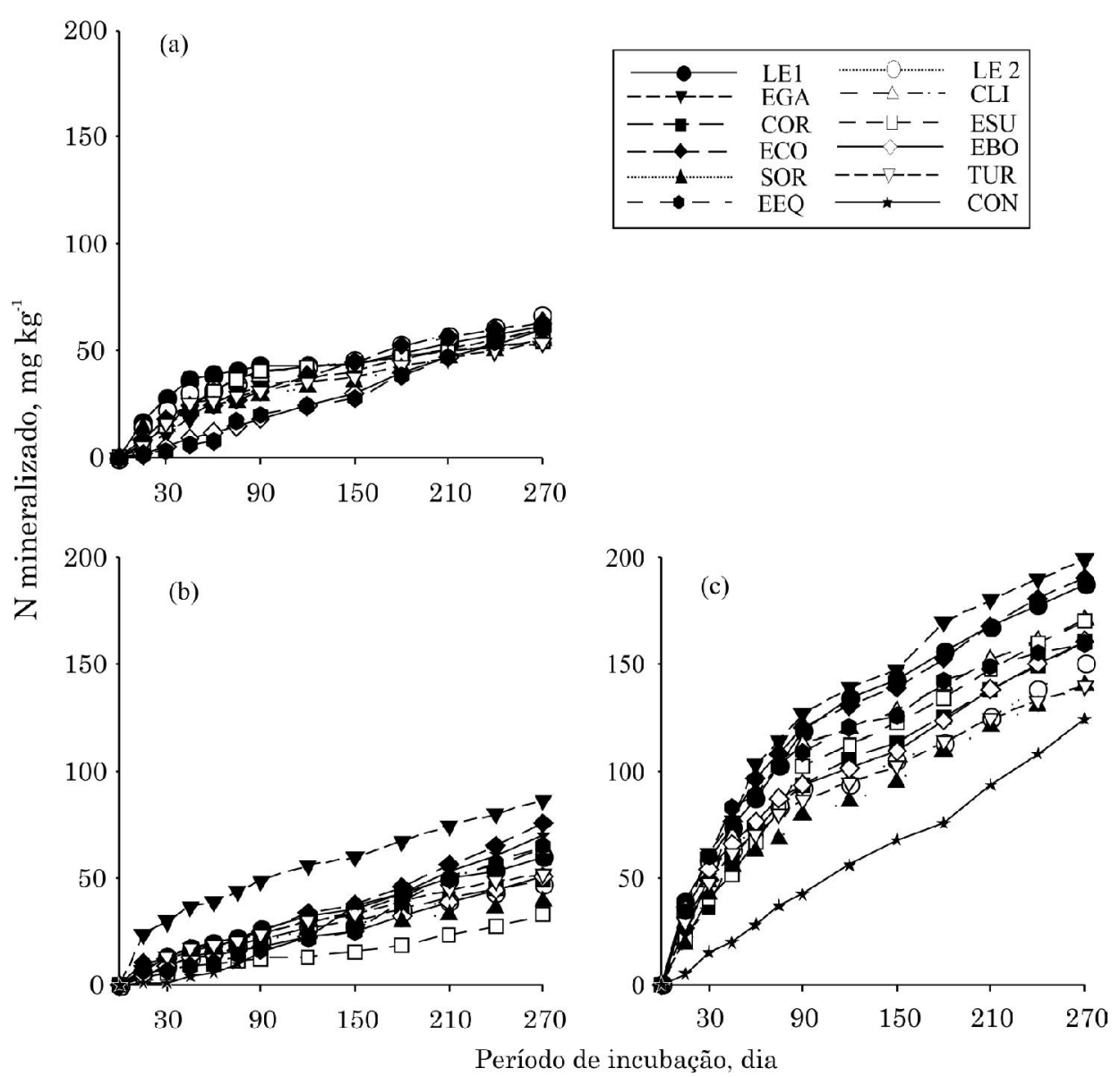

Figura 1. Mineralização acumulada de $\mathbf{N}$ dos resíduos orgânicos incubados com (a) areia lavada, (b) Latossolo Vermelho-Amarelo e (c) Latossolo Vermelho distroférrico, até os 270 dias de incubação. LE 1: lodo de esgoto 1; LE 2: lodo de esgoto 2; EGA: esterco de galinha; CLI: composto de lixo; COR: composto orgânico; ESU: esterco de suíno; ECO: esterco de codorna; EBO: esterco de bovino; SOR: substrato orgânico; TUR: turfa; EEQ: esterco de equino; e CON: controle (sem adição de resíduo).

para outra (Quadro 3). De fato, no LVA, só foi notada mineralização líquida de $\mathrm{N}$ para os tratamentos em que os estercos de galinha e codorna foram adicionados ao solo. Nesse solo, nos demais tratamentos, foi verificada imobilização líquida de $\mathrm{N}$, ou seja, as quantidades de $\mathrm{N}$ mineralizadas pela maioria dos resíduos foram inferiores à notada para o tratamentocontrole. A quantidade imobilizada de $\mathrm{N}$ no LVA variou de um resíduo para outro, sendo as maiores imobilizações líquidas de $\mathrm{N}$ verificadas para $\mathrm{o}$ substrato orgânico e esterco de suíno. Em parte, a imobilização líquida (Quadro 3) de N observada para a maioria dos resíduos incubados no LVA ocorreu, possivelmente, em razão dos baixos teores de $\mathrm{N}$ presentes em alguns dos resíduos orgânicos avaliados, aliado ao baixo teor de matéria orgânica presente nesse solo. Segundo Pottker \& Tedesco (1979), o teor de matéria orgânica regula a mineralização de nutrientes no solo; o teor de $\mathrm{C}$ do solo condiciona, ainda, o $\mathrm{N}$ potencialmente mineralizável, o mesmo ocorrendo com o teor de argila (Rhoden et al., 2006). Em relação ao LVA, é bastante provável que a maior presença de matéria orgânica e o maior teor de argila no LVdf tenham assegurado melhores condições para os organismos mineralizadores de $\mathrm{N}$, e isso, dentre outros fatores, explica a maior velocidade de mineralização de $\mathrm{N}$ pelos resíduos incubados nesse solo.

No LVdf, foi notada mineralização líquida de $\mathrm{N}$, tendo em vista que o $\mathrm{N}$ mineral disponibilizado pelos resíduos orgânicos superou o mineralizado no controle, sendo verificada variação na liberação líquida de $\mathrm{N}$, na faixa de 16 a $75,2 \mathrm{mg} \mathrm{kg}^{-1}$. No LVdf, as menores mineralizações líquidas de $\mathrm{N}$ foram verificadas para a turfa e para o substrato orgânico. Aparentemente, não se observou imobilização líquida de $\mathrm{N}$ no LVdf, mas as quantidades líquidas de $\mathrm{N}$ mineralizado, constatadas para esse solo, para a maioria dos resíduos analisados, são inferiores às notadas para o mesmo resíduo incubado com a areia. Isso sinaliza que, no LVdf, os resíduos incubados não expressam seus potenciais máximos de mineralização e que, se esses potenciais são materializados, há, em longo prazo, imobilização de N no LVdf, quando se considera o 


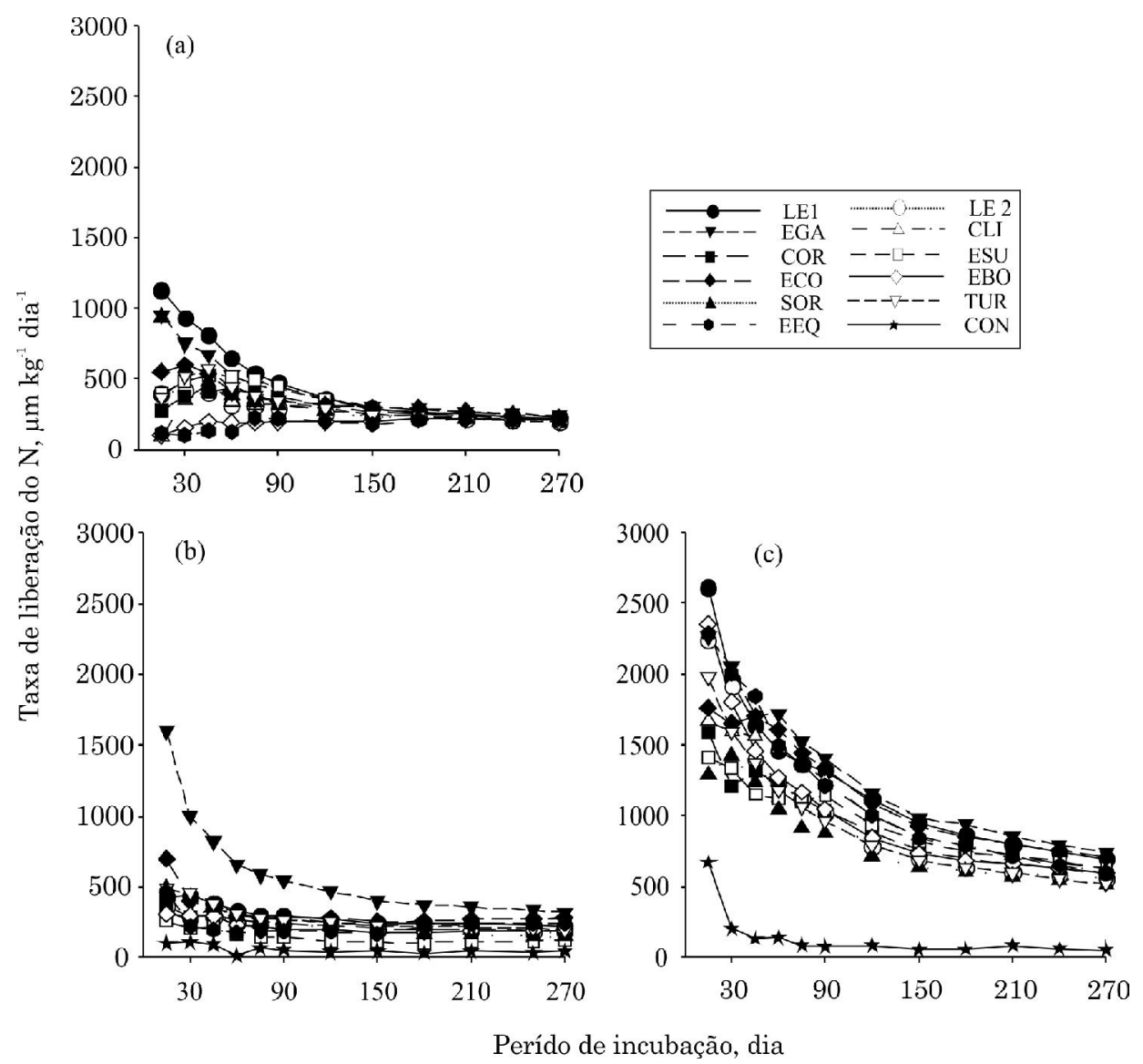

Figura 2. Taxas médias de mineralização diária do $\mathbf{N}$ dos resíduos orgânicos incubados com (a) Areia, (b) Latossolo Vermelho-Amarelo e (c) Latossolo Vermelho distroférrico, até os 270 dias de incubação. LE 1: lodo de esgoto 1; LE 2: lodo de esgoto 2; EGA: esterco de galinha; CLI: composto de lixo; COR: composto orgânico; ESU: esterco de suíno; ECO: esterco de codorna; EBO: esterco de bovino; SOR: substrato orgânico; TUR: turfa; EEQ: esterco de equino; e CON: controle (sem adição de resíduo).

$\mathrm{N}$ mineral disponibilizado pelos resíduos nesse solo, em relação ao da areia. A expressão do potencial de mineralização do $\mathrm{N}$ pelos resíduos incubados com areia pode ser explicada, entre outros fatores, em razão da sua reduzida superfície específica e densidade de carga superficial, fazendo com que essa fração apresente pouco ou nenhum material orgânico fortemente ligado, o que facilita 0 ataque microbiano, resultando, assim, em maior mineralização dos resíduos (Roscoe \& Machado, 2002). Nendel et al. (2005), ao estudarem a mineralização de $\mathrm{N}$ por compostos orgânicos, verificaram que, em dois anos, a porcentagem de $\mathrm{N}$ total mineralizada variou entre 2 e $45,2 \%$, evidenciando-se dependente, entre outros fatores, da textura e do teor de matéria orgânica do solo em que o resíduo orgânico foi adicionado. No trabalho de Khalil et al. (2005), foi notada correlação negativa entre o teor de argila do solo e a mineralização líquida de N. Neste estudo, os dados obtidos estão em desacordo aos obtidos por Khalil et al. (2005), tendo em vista que foi observada correlação inversa entre o $\mathrm{N}$ mineralizado acumulado após 270 dias com o teor de areia do meio de incubação, com $R^{2}$ variando de 0,51 a 0,78 e com coeficiente de correlação médio de $-0,79$, para os 11 resíduos estudados.

$\mathrm{O}$ tempo de meia-vida do $\mathrm{N}$ mineralizado variou de um resíduo orgânico para outro e apresentou-se dependente do solo onde os materiais orgânicos foram incubados (Figura 3). Os valores de $\mathrm{t}^{1} / 2$ observados para os resíduos incubados no LVA foram superiores estatisticamente aos verificados para a areia e LVdf, para a maioria dos resíduos estudados. Os tempos de meia-vida do $\mathrm{N}$ observados para os resíduos incubados com o LVA foram altos, dentro da faixa de 67 a 396 dias. Esses resultados estão relacionados, provavelmente, à imobilização do $\mathrm{N}$ durante a incubação, que foi também verificada no trabalho de Carneiro et al. (2007), que, em ensaio de incubação em condições controladas, averiguaram intensa imobilização do N para resíduos orgânicos. Esses autores observaram que, ao final de 443 dias, lamas celulósicas incubadas disponibilizaram $-43 \%$ do $\mathrm{N}$ total incorporado. Para os resíduos incubados com areia, os tempos de meia-vida variaram de 32 a 129 dias. No trabalho de Espindola et al. (2006), os tempos 
Quadro 3. N mineralizado líquido e teores de N-nitrato e $\mathbf{N}$-amônio até os 270 dias de incubação dos resíduos orgânicos, em areia e em amostras de um Latossolo Vermelho-Amarelo (LVA) e de um Latossolo Vermelho distroférrico (LVdf)

\begin{tabular}{|c|c|c|c|c|c|c|c|c|c|}
\hline \multirow{3}{*}{ Resíduo } & \multicolumn{3}{|c|}{ N mineralizado líquido ${ }^{(1)}$} & \multicolumn{6}{|c|}{ N mineralizado na forma de nitrato ou amônio } \\
\hline & \multirow{2}{*}{ Areia } & \multirow{2}{*}{ LVA } & \multirow{2}{*}{ LVdf } & \multicolumn{2}{|c|}{ Areia } & \multicolumn{2}{|c|}{ LVA } & \multicolumn{2}{|c|}{ LVdf } \\
\hline & & & & $\mathrm{N}-\mathrm{NH}_{4}{ }^{+}$ & $\mathrm{N}-\mathrm{NO}_{3}^{-}$ & $\mathrm{N}-\mathrm{NH}_{4}{ }^{+}$ & $\mathrm{N}-\mathrm{NO}_{3}^{-}$ & $\mathrm{N}-\mathrm{N}_{4}^{+}$ & $\mathrm{N}-\mathrm{NO}_{3}$ \\
\hline & & & & & $\mathrm{mg} \mathrm{kg}^{-1}$ & & & & \\
\hline Lodo de esgoto 1 & $61,4 \mathrm{a}^{(2)}$ & $-10,5 \mathrm{c}$ & $+63,5 \mathrm{a}$ & $34,3 \mathrm{a} * *$ & $27,1 \mathrm{c}$ & $19,9 \mathrm{~b}$ & $40,0 \mathrm{c}$ & $53,5 \mathrm{c}$ & $134 \mathrm{a}$ \\
\hline Lodo de esgoto 2 & 54,8 a & $-22,9 \mathrm{~d}$ & $+26,3 \mathrm{~b}$ & $9,2 \mathrm{~d}$ & $45,6 \mathrm{~b}$ & $9,3 \mathrm{e}$ & $38,2 \mathrm{c}$ & $49,3 \mathrm{c}$ & $101 \mathrm{~b}$ \\
\hline Esterco de galinha & $66,4 \mathrm{a}$ & $+16,6$ a & $+75,2$ a & $26,5 \mathrm{~b}$ & $39,9 \mathrm{~b}$ & 35,4 a & $51,6 \mathrm{~b}$ & $63,9 \mathrm{~b}$ & $135,3 \mathrm{a}$ \\
\hline Composto de lixo & 56,3 a & $-6,6 \mathrm{c}$ & $+46,5 \mathrm{~b}$ & $14,0 \mathrm{c}$ & $42,3 \mathrm{~b}$ & $11,6 \mathrm{~d}$ & $52,2 \mathrm{~b}$ & $44,5 \mathrm{~d}$ & $126 \mathrm{a}$ \\
\hline Composto orgânico & 59,9 a & $-20,8 d$ & $+36,6 \mathrm{~b}$ & $17,4 \mathrm{c}$ & $42,5 \mathrm{~b}$ & $7,6 \mathrm{e}$ & $42,0 \mathrm{c}$ & 33,6 e & $127 \mathrm{a}$ \\
\hline Esterco de suíno & 54,6 a & $-37,4 \mathrm{e}$ & $+46,0 \mathrm{~b}$ & $31,9 \mathrm{a}$ & $22,7 \mathrm{c}$ & $5,6 \mathrm{f}$ & $27,6 \mathrm{~d}$ & $34,7 \mathrm{e}$ & $135,3 \mathrm{a}$ \\
\hline Esterco de codorna & $63,4 \mathrm{a}$ & $+5,6 \mathrm{~b}$ & $+66,1 \mathrm{a}$ & $20,7 \mathrm{~b}$ & $42,7 \mathrm{~b}$ & $13,1 \mathrm{c}$ & 62,9 a & $56,6 \mathrm{c}$ & 133,5 a \\
\hline Esterco de bovino & $60,4 \mathrm{a}$ & $-19,6 \mathrm{~d}$ & $+36,9 \mathrm{~b}$ & $10,9 \mathrm{~d}$ & 49,5 a & $8,3 \mathrm{e}$ & $42,5 \mathrm{c}$ & 68,9 a & $92 \mathrm{~b}$ \\
\hline Substrato orgânico & $55,3 \mathrm{a}$ & $-31,1 \mathrm{e}$ & $+16,3 \mathrm{~b}$ & $11,5 \mathrm{~d}$ & $43,8 \mathrm{~b}$ & $6,7 \mathrm{f}$ & $32,6 \mathrm{~d}$ & $18,9 \mathrm{e}$ & $122 \mathrm{a}$ \\
\hline Turfa & 53,8 a & $-18,2 \mathrm{~d}$ & $+16,0 \mathrm{~b}$ & $25,3 \mathrm{~b}$ & $28,5 \mathrm{c}$ & $11,2 \mathrm{~d}$ & $41,0 \mathrm{c}$ & $54,3 \mathrm{c}$ & $86 \mathrm{~b}$ \\
\hline Esterco de equino & 60,6 a & $-5,2 \mathrm{c}$ & $+35,7 \mathrm{~b}$ & $7,6 \mathrm{e}$ & 53,0 a & $6,9 \mathrm{e}$ & $58,3 \mathrm{~b}$ & $27,7 \mathrm{e}$ & $132 \mathrm{a}$ \\
\hline Controle & - & - & - & - & - & $6,1 \mathrm{f}$ & $64,3 \mathrm{a}$ & $20,6 \mathrm{e}$ & $103,4 \mathrm{~b}$ \\
\hline
\end{tabular}

(1) $\mathrm{N}$ mineralizado líquido ( $\mathrm{N}$ mineralizado na mistura resíduo-solo - $\mathrm{N}$ mineralizado no solo incubado sem resíduo); ${ }^{(2)}$ Médias de teores de $\mathrm{N}$ mineralizado líquido, $\mathrm{N}$-nitrato e $\mathrm{N}$-amônio para os resíduos orgânicos, seguidas de mesma letra, dentro de um mesmo solo ou da areia, não diferem entre si pelo teste de Skott- Knott, a $5 \%$.

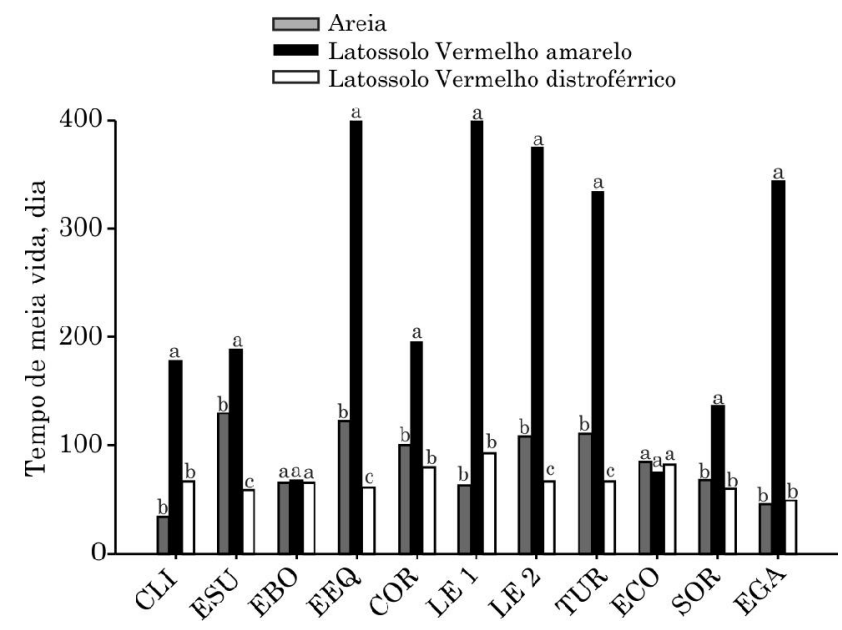

Figura 3. Tempo de meia-vida dos resíduos orgânicos incubados por 270 dias de incubação, em misturas com areia e de Latossolo Vermelho-Amarelo e de Latossolo Vermelho distroférrico. LE 1: lodo de esgoto 1; LE 2: lodo de esgoto 2; EGA: esterco de galinha; CLI: composto de lixo; COR: composto orgânico; ESU: esterco de suíno; ECO: esterco de codorna; EBO: esterco de bovino; SOR: substrato orgânico; TUR: turfa; EEQ: esterco de equino. Médias de tempos de meia-vida do $\mathbf{N}$ mineralizado em Latossolos e em areia, dentro de cada resíduo orgânico, seguidas pela mesma letra não diferem entre si pelo teste de Skott- Knott a $5 \%$. de meia-vida do $\mathrm{N}$ para leguminosas consorciadas com bananeira variaram na faixa de 44 a 110 dias. Ao estimarem a mineralização de $\mathrm{N}$ por plantas de cobertura, Gama-Rodrigues et al. (2007) encontraram tempos de meia-vida de $\mathrm{N}$ na faixa de 43 a 154 dias.

Em relação às formas de $\mathrm{N}$ mineralizado disponibilizadas nos solos e na areia, predominou o $\mathrm{N}$-nitrato, em relação o $\mathrm{N}$-amônio; contudo, as proporções de $\mathrm{N}$-nítrico/N-amoniacal variaram conforme a mistura solo-resíduo analisada (Quadro 3). No LVdf, as condições favoráveis à mineralização de $\mathrm{N}$ também resultaram em maior nitrificação, com teores de N-nitrato, após 270 dias de incubação, variando de 86 a $135,3 \mathrm{mg} \mathrm{kg}^{-1}$; no LVdf, o N-nitrato representou de 2 (lodo de esgoto 2) a 6,6 (substrato orgânico) vezes os teores de $\mathrm{N}$-amônio determinado ao término da incubação. Na areia, à exceção do lodo de esgoto 1 e do esterco de suíno e para os resíduos incubados no LVA, o nitrato predominou em relação ao amônio, mas, proporcionalmente, a disponibilidade de $\mathrm{N}$-nítrico em relação ao amoniacal é menor nesses dois meios, em relação à verificada para os resíduos incubados no LVdf. No trabalho de Boeira \& Maximiliano (2009), a maioria do amônio produzido durante a decomposição de lodos em solo foi convertida em nitrato, de modo que foi verificado aumento linear de N-nítrico ao longo da incubação; seus teores se evidenciaram dependentes da dose de lodo aplicada.

O efeito da composição química dos resíduos orgânicos sobre a mineralização ficou evidenciado quando se avaliou o grau de associação entre as 
quantidades mineralizadas de $\mathrm{N}$ até os 270 dias com os teores de $\mathrm{N}$ total, CSA, C-lábil e relação $\mathrm{C} / \mathrm{N}$ dos resíduos (Quadro 4). A análise dos dados apresentou que o $\mathrm{N}$ mineralizado é regulado pelos teores de $\mathrm{N}$ total e de carbono solúvel em água dos resíduos. Não houve correlação entre o $\mathrm{N}$ mineralizado com a relação $\mathrm{C} / \mathrm{N}$. Neste estudo, a relação $\mathrm{C} / \mathrm{N}$ dos resíduos variou de 4,2/1 (esterco de galinha) a 52,2/1 (substrato orgânico), com a maioria dos resíduos apresentando relação C/N inferior a 15/1. Segundo Bernal et al. (1998), a relação C/N menor que 15/1 implicou em riqueza de $\mathrm{N}$ e maior suscetibilidade do resíduo à decomposição. Em contraponto a isso, relação $\mathrm{C} / \mathrm{N}$ em torno de $10-12 / 1$ pode ser consequência da decomposição dos resíduos pelos organismos do solo, o que acarreta maior grau de humificação e menor suscetibilidade do resíduo ao ataque microbiano. Segundo Cabrera et al. (2005), a relação $\mathrm{C} / \mathrm{N}$, por si só, não explicou as diferenças na mineralização do $\mathrm{N}$, sendo necessário, para isso, que compostos ou grupos de compostos presentes nos resíduos orgânicos sejam identificados. Em razão do que foi dito, não seria aconselhável utilizar a relação $\mathrm{C} / \mathrm{N}$ como único preditor da mineralização de $\mathrm{N}$ pelos resíduos, de modo que o uso desse atributo com essa finalidade deveria ser conjugado com o teor e com as formas de $\mathrm{N}$ presentes nos resíduos, ou com qualquer outro atributo que represente o grau de labibilidade dos materiais orgânicos. A influência da composição química (teor de $\mathrm{N}$, teor de lignina, teor de polifenóis, relação $\mathrm{C} / \mathrm{N}$ etc.) dos resíduos orgânicos tem sido, em diversos estudos, relacionada ao potencial de mineralização do N (Ayuso et al., 1996; Aita \& Giacomini, 2003). No trabalho de Boeira et al. (2002), o acúmulo de $\mathrm{N}$ mineral no solo ao término da incubação apresentou-se dependente dos aportes deN orgânico pelos lodos de esgoto. De acordo com Monteiro et al, (2002), em resíduos de gramíneas e leguminosas, 0 aumento do teor de $\mathrm{C}$ facilmente degradável resulta em mineralização líquida de N. Segundo Aita \& Giacomini (2003), a velocidade de mineralização de N de resíduos de plantas de cobertura evidenciou-se diretamente dependente das concentrações de $\mathrm{N}$ total na fitomassa e de CSA. No trabalho de Melo et al. (2008a), o teor de C-lábil foi considerado inadequado para avaliar o grau de maturação de resíduos orgânicos e apresentou baixa correlação com os teores

Quadro 4. Coeficientes de correlação linear simples entre as quantidades de $\mathbf{N}$ mineralizado até os 270 dias de incubação com algumas características dos resíduos orgânicos

\begin{tabular}{lccccc}
\hline & CSA & N total & C total & C-lábil & C/N \\
\hline LVA & $0,83^{*}$ & $0,83^{*}$ & $0,13^{\mathrm{ns}}$ & $-0,23^{\mathrm{ns}}$ & $-0,44^{\mathrm{ns}}$ \\
LVdf & $0,78^{*}$ & $0,93^{*}$ & $0,13^{\mathrm{ns}}$ & $0,21^{\mathrm{ns}}$ & $-0,56^{\mathrm{ns}}$ \\
Areia & $0,86^{*}$ & $0,75^{*}$ & $0,22^{\mathrm{ns}}$ & $0,16^{\mathrm{ns}}$ & $-0,33^{\mathrm{ns}}$ \\
\hline
\end{tabular}

CSA: carbono solúvel em água; e C-lábil: carbono lábil. * significativo a $1 \%$; e ns: não significativo. de CSA. Com base nisso, neste estudo, a não correlação do $\mathrm{N}$ mineralizado com o C-lábil, possivelmente, indicou que esse atributo não refletiu o grau de humificação dos resíduos, não sendo adequado utilizá-lo para se avaliar a suscetibilidade do resíduo à mineralização, como foi verificado para a relação $\mathrm{C} / \mathrm{N}$.

\section{CONCLUSÕES}

1. A maior velocidade de mineralização de $\mathrm{N}$ ocorreu aos 30-90 dias do período inicial de incubação, sendo notada, após 90-210 dias de incubação, estabilização das taxas de mineralização.

2. O N mineralizado pelos resíduos orgânicos após 270 dias de incubação variou de 33 a $199,2 \mathrm{mg} \mathrm{kg}^{-1}$. Independentemente do Latossolo analisado, os estercos de galinha e codorna são os que propiciaram maiores teores de $\mathrm{N}$ mineralizado. A turfa, o substrato orgânico e o lodo de esgoto 2, em geral, são os resíduos que disponibilizaram em solo menores teores de $\mathrm{N}$ mineral.

3. A mineralização líquida de $\mathrm{N}$, no LVdf, e a imobilização líquida de N, no LVA, foram observadas para a maioria dos resíduos orgânicos, descontados os teores de $\mathrm{N}$ mineralizado no solo sem adição de resíduo (controle).

4. O N mineralizado predominou no solo na forma de nitrato, sendo a proporção de $\mathrm{N}$-nitríco/Namoniacal dependente da mistura solo-resíduo estudada.

5. Os teores de $\mathrm{N}$ total e de carbono solúvel em água dos resíduos orgânicos puderam ser utilizados como preditores do $\mathrm{N}$ mineralizado até os 270 dias de incubação.

\section{AGRADECIMENTOS}

À Fundação de Amparo à Pesquisa do Estado de Minas Gerais (FAPEMIG - processo CAG 1776/06) e ao Conselho Nacional de Desenvolvimento Científico e Tecnológico (CNPq - processos: 302087/2008-7 e 574921/2008-7), pela concessão da bolsa de produtividade em pesquisa a um dos autores e pelo financiamento da pesquisa; e ao Dr. Leônidas Carrijo A. Melo, pelo fornecimento das amostras de resíduos utilizadas neste estudo.

\section{LITERATURA CITADA}

ABREU JUNIOR, C.H.; MURAOKA, T. \& OLIVEIRA, F.C. Carbono, nitrogênio, fósforo e enxofre em solos tratados com composto de lixo urbano. R. Bras. Ci. Solo, 26:769780, 2002. 
AITA, C. \& GIACOMINI, S.J. Matéria orgânica do solo, nitrogênio e enxofre de diversos sistemas de exploração agrícola. In: YAMADA, T.; STIPP, S.R. \& VITTI, A.G.C., orgs. Nitrogênio e enxofre na agricultura brasileira. Piracicaba, SP: International Plant Nutrition Institute, 2007. Cap. 1, p.2-41.

AITA, C. \& GIACOMINI, S.J. Decomposição e mineralização de nitrogênio de resíduos culturais de plantas de cobertura do solo solteiras e consorciadas. R. Bras. Ci. Solo, 28:739$749,2003$.

ALCÂNTARA, M.A.K.; AQUINO NETO, V.; CAMARGO, O.A. \& CANTARELLA, H. Mineralização do nitrogênio em solos tratados com lodos de curtume. Pesq. Agropec. Bras., 42:547-555, 2007.

ALVES, R.N.; MENEZES, R.S.C.; SALCEDO, I.H. \& PEREIRA, W.E. Relação entre qualidade e liberação de $\mathrm{N}$ por plantas do semiárido usadas como adubo verde. R. Bras. Eng. Agric. Amb., 15:1107-1114, 2011.

AMLINGER, F.; GOTZ, B.; DREHER, P.; GESZTI, J. \& WEISSTEINER, C. Nitrogen in biowaste and yard waste compost: dynamics of mobilization and availability: A review. Eur. J. Soil Biol., 39:107-116, 2003.

AYUSO, M.; PASCUAL, J.A.; GARCÍA, C. \& HERNÁNDEZ, T. Evaluation of urban wastes for agricultural use. Soil Sci. Plant. Nutr., 42:105-111, 1996.

BERNAL, M.P.; SÁNCHES-MONEDERO, M.A.; PAREDES, C. \& ROIG, A. Carbon mineralization from organic wastes at different composting stages during their incubation with soil. Agric. Ecosyst. Environ., 69:175-189, 1998.

BOEIRA, R.C. \& MAXIMILIANO, V.C.B. Mineralização de compostos nitrogenados de lodo de esgoto na quinta aplicação em Latossolo. R. Bras. Ci. Solo, 33:711-722, 2009.

BOEIRA, R.C.; LIGO, M.A.V. \& DYNIA, J.F. Mineralização de nitrogênio em solo tropical tratado com lodos de esgoto. Pesq. Agropec. Bras., 37:1639-1647, 2002.

CABRERA, M.L.; KISSEL, D.E. \& VIGIL, M.F. Nitrogen mineralization from organic residues: Research opportunities. J. Environ. Qual., 34:75-79, 2005.

CARNEIRO, J.P.; BRANCO, S. \& COUTINHO, J. Mineralização de azoto de diferentes resíduos orgânicos em incubação laboratorial de longa duração. R. Ci. Agr., 30:159-173, 2007.

COSTA, F.X.; SEVERINO, S.L.; BELTRÃO, N.E.M.; FREIRE, R.M.; LUCENA, A.M.A. \& GUIMARÃES, M.M.B. Avaliação de teores químicos na torta de mamona. R. Biol. Ci. Terra, 4:132-144, 2004.

DAR, G.H. \& MISHRA, M.M. Influence of cadmium on carbon and nitrogen mineralization in sewage-sludge amended soils. Environ. Pollut., 84:285-290, 1994.

EGHBALL, B.; WIENHOLD, B.J.; GILLEY, J.E. \& EIGENBERG, R.A. Mineralization of manure nutrients. J. Soil Water Conserv., 57:470-473, 2002.

ESPINDOLA, J.A.A.; GUERREIRA, D.L.A.; TEIXEIRA, M.G. \& URQUIAGA, S. Decomposição e liberação de nutrientes acumulados em leguminosas herbáceas perenes consorciadas com bananeira. R. Bras. Ci. Solo, 30:321-328, 2006.
FERREIRA, D.F. SISVAR. Lavras, Universidade Federal de Lavras, 2003.

GAMA-RODRIGUES, A.C.; GAMA-RODRIGUES, E.F. \& BRITO, E.C. Decomposição e liberação de nutrientes de resíduos culturais de plantas de cobertura em Argissolo Vermelho-Amarelo na região noroeste fluminense-RJ. R. Bras. Ci. Solo, 31:1421-1428, 2007.

GIACOMINI, S.J. \& AITA, C. Decomposição e liberação de nitrogênio dos resíduos culturais de plantas de cobertura de solo solteiras e consorciadas. R. Bras. Ci. Solo, 27:601$612,2003$.

HIGASHIKAWA, F.S.; SILVA, C.A. \& BETTIOL, W. Chemical and physical properties of organic residues. R. Bras. Ci. Solo, 34:1743-1752, 2010.

JANZEN, H.H. \& KUCEY, R.M.N. C, N, and S mineralization of crop residues as influenced by crop species and nutrient regime. Plant Soil, 106:35-41, 1988.

KHALIL, M.I.; HOSSAIN, M.B. \& SCHMIDHALTER, U. Carbon and nitrogen mineralization in different soils of the subtropics treated with organic materials. Soil. Biol. Biochem., 37:1507-1518, 2005.

KHAN, M. \& SCULLION, J. Effects of metal $(\mathrm{Cd}, \mathrm{Cu}, \mathrm{Ni}, \mathrm{Pb}$, $\mathrm{Zn}$ ) enrichment on sewage-sludge on soil microorganisms and their activities. Appl. Soil Ecol., 20:145$155,2002$.

KIEHL, E.J. Fertilizantes orgânicos. São Paulo, Agronômica Ceres, 1985. 44p.

MANTOVANI, J.R., FERREIRA, M.E.; CRUZ, M.C.P.; BARBOSA, J.C.; FERREIRA, A.C. Mineralização de carbono e de nitrogênio provenientes de composto de lixo urbano em argissolo. R. Bras. Ci. Solo, 30:677-684, 2006.

MELO, L.C.A.; SILVA, C.A. \& DIAS, B.O. Caracterização da matriz orgânica de resíduos de origens diversificadas. $R$. Bras. Ci. Solo, 32:101-110, 2008a.

MELO, L.C.A.; SILVA, C.A. \& DIAS, B.O. Influência de métodos de digestão e massa de amostra na recuperação de nutrientes em resíduos orgânicos. Quím. Nova, 31:556-561, 2008 b.

MONTEIRO, H.C.F.; CANTARUTTI, R.B.; NASCIMENTO JUNIOR, D.; REGAZZI, A.J. \& FONSECA, D.M. Dinâmica de decomposição e mineralização de nitrogênio em função da qualidade de resíduos de gramíneas e leguminosas forrageiras. R. Bras. Zootec., 31:1092-1102, 2002.

NENDEL, C.; REUTER, S.; KERSEBAUM, K.C.; KUBIAK, R. $\&$ NIEDER, R. Nitrogen mineralization from mature biowaste compost in vineyard soils II. Test of $\mathrm{N}$ mineralization parameters in a long-term in situ incubation experiment. J. Plant Nutr. Soil Sci., 168:219-227, 2005.

PANSU, M. \& THURIÈS, L. Kinetics of $\mathrm{C}$ and $\mathrm{N}$ mineralization, $\mathrm{N}$ immobilization and $\mathrm{N}$ volatilization of organic inputs in soil. Soil Biol. Biochem., 35:37-48, 2003.

PAUL, E.A. \& CLARK, F.E. Soil microbiology and biochemistry. New York, Academic Press, 1996. 340p.

PEREIRA, J.M.; MUNIZ, J.A. \& SILVA, C.A. Nonlinear models to predict nitrogen mineralization in an Oxisol. Sci. Agric., 62:395-400, 2005. 
POTTKER, D. \& TEDESCO, M.J. Efeito do tipo e tempo de incubação sobre a mineralização da matéria orgânica e nitrogênio total em solos do Rio Grande do Sul. R. Bras. Ci. Solo, 3:20-24, 1979.

RHODEN, A.C.; SILVA, L.S.; CAMARGO, F.A.O.; BRITZKE, D. \& BENEDETTI, E.B. Mineralização anaeróbia do nitrogênio em solos de várzea do Rio Grande do Sul. Ci. Rural, 36:1780-1787, 2006.

ROSCOE, R. \& MACHADO, P.L.O.A. Fracionamento físico do solo em estudos da matéria orgânica. Dourados, Embrapa agropecuária Oeste/Rio de Janeiro, Embrapa Solos, 2002. 86p.

SCHOMBERG, H.H.; WIETHOLTER, S.; GRIFFIN, T.S.; REEVES, D.W.; CABRERA, M.L.; FISHER, D.S.; ENDALE, D.M.; NOVAK, J.M.; BALKCOM, K.S.; RAPER, R.L.; KITCHEN, N.R.; LOCKE, M.A.; POTTER, K.N.; SCHWARTZ, R.C.; TRUMAN, C.C. \& TYLER, D.D. Assessing indices for predicting potential nitrogen mineralization in soils under different management systems. Soil Sci. Soc. Am. J., 73:1575-1586, 2009.
SILVA, F.C., org. Manual de análises químicas de solos, plantas e fertilizantes. Brasília, Embrapa Comunicação para Transferência de Tecnologia, 1999. 370p.

STANFORD, G. \& SMITH, S.J. Nitrogen mineralization potentials of soils. Soil Sci. Soc. Am. J., 36:465-472, 1972.

TEDESCO, M.J.; GIANELLO, C.; BISSANI, C.A.; BOHNEN, H. \& VOLKWEISS, S.J. Análises de solo, plantas e outros materiais. 2.ed. Porto Alegre, Universidade Federal do Rio Grande do Sul, 1995. 174p. (Boletim Técnico, 5)

TORRES, J.L.R.; PEREIRA, M.G.; ANDRIOLI, I.; POLIDORO, J.C. \& FABIAN, A.J. Decomposição e liberação de nitrogênio de resíduos culturais de plantas de cobertura em um solo de cerrado. R. Bras. Ci. Solo, 29:609-618, 2005.

TRINSOUTROT, I.; RECOUS, S.; BENTZ, B.; LINERES, M.; CHENEBY, D. \& NICOLARDOT, B. Biochemical quality of crop residues and carbon and nitrogen mineralization kinetics under non limiting nitrogen conditions. Soil Sci. Soc. Am. J., 64:918-926, 2000. 